\title{
Otubain 2 is a novel promoter of beta cell survival as revealed by siRNA high-throughput screens of human pancreatic islets
}

\author{
A. Beck • Y. Vinik • H. Shatz-Azoulay • R. Isaac • \\ S. Streim • G. Jona $\cdot$ S. Boura-Halfon • Y. Zick
}

Received: 25 July 2012 / Accepted: 28 February 2013 /Published online: 21 March 2013

(C) Springer-Verlag Berlin Heidelberg 2013

\begin{abstract}
Aims/hypothesis Pro-inflammatory cytokines induce death of beta cells and hamper engraftment of transplanted islet mass. Our aim was to reveal novel genes involved in this process, as a platform for innovative therapeutic approaches.

Methods Small interfering RNA (siRNA) high-throughput screening (HTS) of primary human islets was employed to identify novel genes involved in cytokine-induced beta cell apoptosis. Dispersed human islets from nine human donors, treated with a combination of TNF- $\alpha$, IL- $1 \beta$ and IFN- $\gamma$ were transfected with $\sim 730$ different siRNAs. Caspase-3/7 activity was measured, results were analysed and potential anti- and pro-apoptotic genes were identified.

Results Dispersed human pancreatic islets appeared to be suitable targets for performance of siRNA HTS. Using this methodology we found a number of potential pro- and antiapoptotic target hits that have not been previously associated with pancreatic beta cell death. One such hit was the deubiquitinating enzyme otubain 2 (OTUB2). OTUB2 knockdown increased caspase-3/7 activity in MIN6 cells and primary human islets and inhibited insulin secretion and increased nuclear factor- $\mathrm{kB}$ (NF-kB) activity both under basal conditions and following cytokine treatment.

Conclusions Use of dispersed human islets provides a new platform for functional HTS in a highly physiological system. Employing this technique enabled the identification of OTUB2 as a novel promoter of viability and insulin
\end{abstract}

Electronic supplementary material The online version of this article (doi:10.1007/s00125-013-2889-x) contains peer-reviewed but unedited supplementary material, which is available to authorised users.

A. Beck · Y. Vinik $\cdot$ H. Shatz-Azoulay $\cdot$ R. Isaac $\cdot$ S. Streim

G. Jona $\cdot \mathrm{S}$. Boura-Halfon $\cdot$ Y. Zick $(\triangle)$

Department of Molecular Cell Biology,

Weizmann Institute of Science, 234 Herzl Street,

Rehovot 76100, Israel

e-mail: yehiel.zick@weizmann.ac.il secretion in human beta cells. OTUB2 acts through the inhibition of NF- $\mathrm{KB}$ signalling, which is deleterious to beta cell survival. siRNA screens of human islets may therefore identify new targets, such as OTUB2, for therapeutic intervention in type 1 diabetes and islet transplantation.

Keywords Human islets · OTUB2 · Pro-inflammatory cytokines $\cdot$ siRNA screens

$\begin{array}{ll}\text { Abbreviations } \\ \text { ER } & \text { Endoplasmic reticulum } \\ \text { GSIS } & \text { Glucose-stimulated insulin secretion } \\ \text { HTS } & \text { High-throughput screening } \\ \text { IkB } & \text { Inhibitor of } \mathrm{kB} \\ \text { IKK } & \text { IkB kinase } \\ \text { iNOS } & \text { Inducible nitric oxide synthase } \\ \text { JNK } & \text { c-Jun N-terminal kinase } \\ \text { MAPK } & \text { Mitogen-activated protein kinases } \\ \text { NEMO } & \text { NF-kB essential modulator } \\ \text { NF-kB } & \text { Nuclear factor kappaB } \\ \text { NO } & \text { Nitric oxide } \\ \text { NonT } & \text { Non-targeting } \\ \text { OTUB2 } & \text { Otubain 2 } \\ \text { PI } & \text { Propidium iodide } \\ \text { qRT-PCR } & \text { Quantitative real-time PCR } \\ \text { RIP1 } & \text { Receptor-interacting protein 1 } \\ \text { siRNA } & \text { Small interfering RNA } \\ \text { TAK1 } & \text { TGF-beta activated kinase 1 } \\ \text { TBS } & \text { Tris-buffered saline } \\ \text { TRAF } & \text { TNF receptor-associated factor }\end{array}$

\section{Introduction}

Pancreatic beta cell dysfunction underlies essentially all major forms of diabetes [1]. In type 1 diabetes, cell death 
is caused by an autoimmune assault against beta cells by infiltrating lymphocytes resulting in autoimmune destruction, insulin deficiency and hyperglycaemia [2]. Islet transplantation is a promising therapy for type 1 diabetes, and brings about insulin independence and a regain of normoglycaemia. However, it has been estimated that over $70 \%$ of transplanted islets are destroyed in the early posttransplant period [3]. Local secretion of pro-inflammatory cytokines, such as IL- $1 \beta$, TNF- $\alpha$ and IFN- $\gamma$, by immune cells invading the islets contribute to beta cell demise $[2,4]$ through the activation of a number of signalling pathways [5]. These pathways include: stress-activated protein kinases c-Jun N-terminal kinase (JNK) and p38 mitogen-activated protein kinase (MAPK) [6]; the triggering of an endoplasmic reticulum (ER) stress response and the release of death signals from mitochondria [7, 8]; activation of inducible nitric oxide synthase (iNOS) and release of cytotoxic nitric oxide (NO) $[6,9]$ and activation of the transcription factor nuclear factorkappaB (NF-kB) [10, 11].

$\mathrm{NF}-\mathrm{kB}$ activation is thought to provoke cytokine-induced death of human islets [12-14]. This process is initiated following the phosphorylation of inhibitor of $\mathrm{kB}$ (IKB), which targets it to ubiquitination and subsequent degradation by the proteasome, thus enabling nuclear translocation of NF- $\mathrm{KB}$ [15]. Aside from its role in promoting degradation of elements along the NF- $\mathrm{KB}$ signalling pathway, ubiquitination also serves to regulate the function of a number of proteins along this pathway (e.g. TNF receptorassociated factor $2 / 5$ [TRAF2/5]; receptor-interacting protein 1 [RIP1]), mainly by promoting protein-protein interactions [16]. Conversely, de-ubiquitinating enzymes, such as CYLD and A20, execute an opposite effect, thus restricting NF- $\mathrm{kB}$ activity and inhibiting apoptosis of beta cells $[13,17]$. Still, despite extensive studies, the spectrum of signalling elements that control beta cell death either through the NF- $\mathrm{kB}$ or other pathways remains incompletely understood.

Screening of small interfering RNA (siRNA) libraries has become an increasingly effective method in functional genomics [18], leading to the identification of novel genes involved in diverse cellular processes. To date, the majority of high-throughput screening (HTS) protocols have been established in cell lines due to the need for robustness and homogeneity $[19,20]$. Nonetheless, the use of primary human islets for functional siRNA HTS provides the opportunity to obtain a wide spectrum of data from a highly physiological source. In the current study we employed this novel screening mode in an attempt to reveal new substances involved in cytokine-induced death of human pancreatic islets. Our findings implicate otubain 2 (OTUB2) [21], a de-ubiquitinating enzyme of TRAF3 and TRAF6 [22], as a potent inhibitor of death of human beta cells.

\section{Methods}

Materials Pen/Strep, Hanks' solution, DMEM, CMRL 1066 medium and trypsin-EDTA were supplied by Biological Industries (Beit Haemek, Israel). FBS was from Hyclone Laboratories (Logan, UT, USA). siRNA SMARTpool libraries and transfection reagents were provided by Dharmacon (Lafayette, CO, USA). IL- $1 \beta$ and IFN- $\gamma$ were purchased from MD Biosciences (Ness Ziona, Israel). TNF- $\alpha$ was provided by Prospec-Tany Technogene (Rehovot, Israel). Protein-G agarose beads, TRAF6 and OTUB2 antibodies were from Santa Cruz Biotechnology (Santa Cruz, CA, USA). Anti-ubiquitin antibodies were from Covance (Berkeley, CA, USA). Cytokine mixture ( $\mathrm{x}$ cytomix) consisted of $3 \mathrm{nmol} / 1 \mathrm{TNF}-\alpha, 3 \mathrm{nmol} / 1 \mathrm{IFN}-\gamma$ and $1.5 \mathrm{nmol} / 1 \mathrm{IL}-1 \beta$ (biological activity: $10 \mathrm{U} / \mathrm{ng}$ [TNF- $\alpha$, IFN- $\gamma]$ and $200 \mathrm{U} / \mathrm{ng}$ [IL-1 $\beta])$. In certain experiments two- and threefold higher concentrations of cytokine mixtures were used. These were referred to as $2 \mathrm{x}$ - and $3 \mathrm{x}$-cytomix, respectively.

Culture of human islets Isolated human islets ( $>90 \%$ purity, confirmed by dithizone staining) were provided through the JDRF award 31-2008-413 (ECIT Islet for Basic Research program). Islets were cultured in CMRL 1066 medium containing 10\% FBS, $2 \mathrm{mmol} / 1 \mathrm{l}$-glutamine, $100 \mathrm{U} / \mathrm{ml}$ penicillin, $100 \mu \mathrm{g} / \mathrm{ml}$ streptomycin, $0.25 \mu \mathrm{g} / \mathrm{ml}$ amphotericin and $40 \mu \mathrm{g} / \mathrm{ml}$ gentamicin at $37^{\circ} \mathrm{C}$ in a $5 \% \mathrm{CO}_{2}$ humidified atmosphere. The medium was changed every other day. Human islets were dispersed following a 4 min incubation at $37^{\circ} \mathrm{C}$ with trypsin-EDTA. Trypsinised islets were washed with cold CMRL 1066 medium containing 10\% FBS, gently pipetted and resuspended in CMRL 1066 containing 10\% FBS. Cells were used within $60 \mathrm{~h}$ following dispersion. Human islets studies received Ethics Committee approval.

Cells MIN6 cells (passages 24-40) were cultured in DMEM containing $11 \mathrm{mmol} / \mathrm{l}$ glucose supplemented with $10 \% \mathrm{FBS}$, $2 \mathrm{mmol} / \mathrm{l}$ L-glutamine, $5 \mu \mathrm{mol} / 1 \beta$-mercaptoethanol, $100 \mathrm{U} / \mathrm{ml}$ penicillin and $100 \mu \mathrm{g} / \mathrm{ml}$ streptomycin. HEK293 cells were cultured in DMEM supplemented with 5\% FBS, $2 \mathrm{mmol} / \mathrm{l}$ L-glutamine, $100 \mathrm{U} / \mathrm{ml}$ penicillin and $100 \mu \mathrm{g} / \mathrm{ml}$ streptomycin. Cells were grown at $37^{\circ} \mathrm{C}$ in a $5 \% \mathrm{CO}_{2}$ humidified atmosphere.

High throughput screening HTS was carried out on human islets derived from nine patients, essentially as described previously [23], using 730 non-redundant human siRNA SMARTpools, purchased from Dharmacon. Each SMARTpool contained four different siRNA duplexes targeting a particular mRNA. Aliquots of each siRNA SMARTpool were transferred into 384-well plates to reach a final siRNA concentration of $25 \mathrm{nmol} / \mathrm{l}$ per well. Scrambled 
sequences of non-targeting (nonT) siRNAs and siRNAs for caspase-3 and TNF receptor served as controls and were present in each plate. Dispersed human islets $(10,000-$ 15,000 cells/well in different experiments) were distributed into the wells using an automated Multidrop 96/384 well micro plate dispenser (MTX Lab Systems, Vienna, VA, USA) and were transiently reverse-transfected with the siRNA SMARTpools using Dharmafect-1 transfection reagent (Dharmacon). Forty-eight hours post-transfection the cells were treated for $24 \mathrm{~h}$ with $3 \mathrm{x}$-cytomix, after which caspase $3 / 7$ activity was determined using the Enzolyte-RH110 caspase-3/7 assay kit (AnaSpec, San Jose, CA, USA) according to the manufacturer's instructions. Fluorescence intensity was measured using an Infinite 200 PRO multimode reader (Tecan Trading, Männedorf, Switzerland) at an excitation/emission wavelength of $485 / 520 \mathrm{~nm}$.

Data analysis was performed independent of gene annotations; readings of each plate were analysed by an ANOVA algorithm, using the Parteck Genomics Suite program (Parteck, St Louis, MI, USA). Measurement of the plate's noise was calculated by dividing the SD of the plate's measurements by its average. On average, the noise of the plates was $\sim 20 \%$. The read-outs of each well were first normalised to obtain the $z$ score by subtracting the plate's average from a given well's value and dividing the result by the plate's SD, to enable plate-to-plate comparisons. Due to the large heterogeneity and variability of primary human islets only $z$ score $>|1.1|$ for a given gene obtained in at least $4 / 9$ patients was selected for further analysis. To enable such selection an $m z$ score harbouring the above criteria was defined and genes having $m z$ scores $\geq 4.4$ were selected as potential hits for further analysis (Table 1).

The $m z$ score was defined as follows: when given $n$ genes, each associated with a k-tuple of numbers called repeats, the set of $\mathrm{k} n$ numbers $\{g(i, j) \mid i=1, \ldots, n, j=1, \ldots, k\}$ is considered. The following constants are used: $L$, the minimal absolute value of $g(i, j)$ for a repeat to be considered (in the present screen $L=1.1$ was used); $\alpha$, the minimal required fraction of the k-tuple of numbers (in the present screen $\alpha=$ $0.4)$. The $m z$ score is therefore defined by several steps:

Define a function $f(i, j)$ by

$f(i, j)=\left\{\begin{array}{cc}1 & \text { if }|g(i, j)| \geq L \\ 0 & \text { otherwise }\end{array}\right.$

thus $f(i, j)$ is 1 if and only if either $g(i, j) \geq L$ or $g(i, j) \leq-L$. Define now

$\operatorname{mzscore}(i)=\left\{\begin{array}{cc}\sum_{j=1}^{k} f(i, j) g(i, j) & \text { if } \sum_{j=1}^{k} f(i, j) \geq \alpha k \\ 0 & \text { otherwise }\end{array}\right.$

meaning that mzscore $(i)$ is the sum of the $\mathrm{k}$ values $g(i, 1)$ to $g(i, k)$ that are associated with the gene indexed $i$, and have an absolute value of at least $L$, but only if there are at least $\alpha \mathrm{k}$ of these $\mathrm{k}$ values with this property. Otherwise, mzscore $(i)$ is defined as 0 .

Assay of caspase-3/7 activity independent of the screen Dispersed human islets $(15,000$ cells/well) were seeded in 384-well plates in $50 \mu \mathrm{l}$ DMEM and were treated as indicated. Caspase-3/7 activity was then assayed in five replicates per treatment as described above.

Assay of cellular reducing power Cellular reducing power in human islets was determined using the CellTiter-Blue (CTB) fluorimetric assay (Promega, Masidon, WI, USA). Fluorescence intensity was measured using an Infinite 200 PRO multimode reader (Tecan Trading) at an excitation/ emission wavelength of $560 \mathrm{~nm} / 590 \mathrm{~nm}$.

$N F-\kappa B$ activity NF-KB activity was determined using the Ready-To-Glow secreted luciferase assay kit (Clontech, Mountain View, CA, USA) according to the manufacturer's instructions. In brief, MIN6 cells were transfected with the indicated siRNAs. At $24 \mathrm{~h}$ post transfection cells were further transfected with $200 \mathrm{ng}$ of a secreted luciferase plasmid, coupled to the NF-KB enhancer elements. Constitutively secreted luciferase plasmid, transfected to separate wells, served as control. Twenty-four hours thereafter cells were treated with $\mathrm{x}$-cytomix for the indicated times, to induce NF- $\mathrm{KB}$ activity. Fifty microlitres of the cells' media were harvested and transferred to black 96-well plates. A luciferase assay mix was added $(5 \mu \mathrm{l} /$ well $)$ and luminescence was read using Infinite 200 PRO multimode reader (Tecan Trading).

Insulin secretion MIN6 cells and primary human islets were incubated for $60 \mathrm{~min}$ with 0.5 or $3.3 \mathrm{mmol} / 1$ glucose, respectively, in KRBH buffer $(124 \mathrm{mmol} / \mathrm{l} \mathrm{NaCl}, 5.6 \mathrm{mmol} / \mathrm{l}$ $\mathrm{KCl}, 2.5 \mathrm{mmol} / \mathrm{l} \mathrm{CaCl}_{2}$ and $20 \mathrm{mmol} / \mathrm{l}$ HEPES pH7.4). Cells were then incubated with 20 or $16.7 \mathrm{mmol} / 1$ glucose in $\mathrm{KRBH}$ buffer for $60 \mathrm{~min}$ at $37^{\circ} \mathrm{C}$. Cellular insulin content and the content of insulin secreted to the medium were determined using mouse or human insulin ELISA kits (Mercodia, Uppsala, Sweden) according to the manufacturer's instructions.

Quantitative real-time PCR For quantitative real-time PCR (qRT-PCR), RNA was extracted using the PerfectPure RNA kit (5Prime, Gaithersburg, MD, USA) and cDNA was generated by cDNA Reverse Transcription kit (Applied Biosystems, Foster City, CA, USA). Quantitative detection of specific mRNA transcripts was carried out by real-time PCR using an ABI-PRISM 7300 instrument (Applied Biosystems) with SYBR green PCR mix (Invitrogen, Grand Island, NY, USA) and the sets of primers listed in the electronic supplementary material (ESM) Methods. 
Table 1 Genes having $m z$ score $\geq 4.4$, as defined in the Methods

\begin{tabular}{|c|c|c|c|c|}
\hline Gene & GenBank accession number & $z$ score $\geq|1.1|$ & $m z$ score & Description \\
\hline \multicolumn{5}{|c|}{ Pro-apoptotic hits } \\
\hline ITCH & NM_031483 & $5 / 9$ & 7.0 & Itchy E3 ubiquitin protein ligase homologue \\
\hline$G P R 92^{\mathrm{a}}$ & NM_001142961 & $5 / 9$ & 6.9 & Lysophosphatidic acid receptor 5 \\
\hline FBXO8 & NM_012180.2 & $4 / 9$ & 7.0 & F-box protein 8 \\
\hline$U B E 2 A$ & NM_003336.2 & $4 / 9$ & 6.0 & Ubiquitin-conjugating enzyme E2A \\
\hline RNF111 & NM_017610.6 & $4 / 8$ & 6.0 & Ring finger protein 111 \\
\hline$D M T F 1$ & NM_001142326.1 & $4 / 9$ & 5.4 & Cyclin D binding myb-like transcription factor 1 \\
\hline$A N K I B 1$ & XM_377955 & $5 / 9$ & 5.2 & Homo sapiens ankyrin repeat and IBR domain containing 1 \\
\hline \multicolumn{5}{|c|}{ Anti-apoptotic hits } \\
\hline$B R A F$ & NM_004333.4 & $4 / 9$ & 5.5 & V-raf murine sarcoma viral oncogene homologue B1 \\
\hline$R F X 5$ & NM_000449.3 & $4 / 9$ & 7.3 & Regulatory factor X, 5 (influences HLA class II expression) \\
\hline RFPL3 & NM_001098535.1 & $4 / 8$ & 6.2 & Ret finger protein-like 3 \\
\hline FLJ14981 & NM_032868 & $4 / 9$ & 6.2 & MPN domain containing \\
\hline$R A B 6 B$ & NM_016577.3 & $4 / 9$ & 6.4 & RAB6B, member RAS oncogene family \\
\hline USP16 & NM_001001992.1 & $4 / 9$ & 6.1 & Ubiquitin specific peptidase 16 \\
\hline ATF3 & NM_001030287.3 & $4 / 9$ & 7.2 & Activating transcription factor 3 \\
\hline$U B E 2 M$ & NM_003969.3 & $4 / 9$ & 7.4 & Ubiquitin-conjugating enzyme E2M \\
\hline PTCH2 & NM_001166292.1 & $4 / 9$ & 7.4 & Patched 2 \\
\hline MARCH3 & NM_001005415.1 & $4 / 9$ & 7.8 & Membrane-associated ring finger $(\mathrm{C} 3 \mathrm{HC} 4) 3$ \\
\hline$M D M 2$ & NM_002392.3 & $4 / 9$ & 7.5 & Mdm2 p53 binding protein homologue \\
\hline USP18 & NM_017414.3 & $4 / 9$ & 8.2 & Ubiquitin specific peptidase 18 \\
\hline$R N F 40$ & NM_001207033.1 & $6 / 9$ & 12.6 & Ring finger protein 40 \\
\hline OTUB2 & NM_023112.3 & $5 / 9$ & 16.6 & OTU domain, ubiquitin aldehyde binding 2 \\
\hline
\end{tabular}

a Also known as LPAR5

${ }^{\mathrm{b}}$ Also known as MPND

Immunoprecipitation Cells were washed three times with PBS and were harvested in buffer A $(25 \mathrm{mmol} / \mathrm{l}$ Tris- $\mathrm{HCl}$, $2 \mathrm{mmol} / 1$ sodium orthovanadate, $0.5 \mathrm{mmol} / 1 \mathrm{EGTA}$, $10 \mathrm{mmol} / 1 \mathrm{NaF}, 10 \mathrm{mmol} / 1$ sodium pyrophosphate, $80 \mathrm{mmol} / \mathrm{l} \beta$-glycerophosphate, $25 \mathrm{mmol} / \mathrm{NaCl}, 1 \%$ Triton X-100, protease inhibitor cocktail diluted 1:1,000, pH7.4). Cell extracts were centrifuged at 20,000 $g$ for $15 \mathrm{~min}$ at $4{ }^{\circ} \mathrm{C}$ and supernatant fractions were immunoprecipitated with the indicated antibodies, resolved by $10 \%$ SDS-PAGE and transferred to nitrocellulose membranes for western blotting as described previously [24].

In vivo ubiquitination MIN6 cells were transiently transfected with the indicated siRNAs. At $72 \mathrm{~h}$ post transfection, cells were washed three times with ice-cold PBS and were lysed in $200 \mu \mathrm{l}$ of $1 \%$ SDS in tris-buffered saline (TBS) $(150 \mathrm{mmol} / \mathrm{l} \mathrm{NaCl}, 10 \mathrm{mmol} / \mathrm{l}$ Tris- $\mathrm{HCl}, \mathrm{pH} 7.5)$ by boiling twice for $5 \mathrm{~min}$ after vigorous vortexing. Next, $400 \mu \mathrm{l}$ of TBS containing $1.5 \%$ Triton X-100 was added to the lysates as we described previously [24]. After centrifugation for $15 \mathrm{~min}$ at $12,000 \mathrm{~g}$ at $24^{\circ} \mathrm{C}$, the supernatant fractions were collected for immunoprecipitation with anti-
TRAF6 antibodies. Samples were resolved by SDS-PAGE and were western blotted with the indicated antibodies.

FACS analysis MIN6 cells were treated as indicated, harvested and rinsed twice with PBS. Cells were stained for propidium iodide (PI) and Annexin-V using the Annexin-V-FLUOS staining kit (Roche Applied Science, Indianapolis, IN, USA) according to the manufacturer's instructions. Flow cytometry was performed on a two-laser, FACSCalibur (BD Biosciences, San Jose, CA, USA). Data were analysed with BD Cellquest Pro software.

Statistical analysis Data are presented as mean \pm SEM. Data were analysed by Student's $t$ test at a minimum $p<0.05$ threshold.

\section{Results}

Dispersion of human islets reduces the noise of highthroughput siRNA screens Intact isolated human pancreatic islets are the most physiological system for siRNAs screens, 
although their heterogeneity in cell number and size makes them a less than an optimal system. To somewhat overcome this problem human islets were dispersed into smaller fragments as described [25], achieving a more homogenous population. To determine the reduction in noise due to dispersion, intact or dispersed human islets were seeded in 384-well plates and caspase-3/7 activity and cellular reducing power were measured. A fourfold and twofold decrease in background noise (calculated as described under Methods) was observed, when caspase-3/7 activity (Fig. 1a) or cellular reducing power (Fig. 1b) was assayed, respectively, using dispersed instead of intact islets. These findings suggest that islet dispersion better homogenises the cell population (Fig. 1c) and reduces background noise, thus rendering the system more appropriate for HTS.

Optimising conditions for siRNA HTS using cytokine-treated dispersed human islets To optimise the conditions required to perform siRNA HTS, time course and dose-response experiments were carried out using cells treated with a mixture of cytokines. A threefold increase in caspase-3/7 activity was detected when dispersed human islets were treated with 3x-cytomix for $24 \mathrm{~h}$ (ESM Fig. 1a), while lower concentrations (e.g. x-cytomix, 2x-cytomix) augmented caspase-3/7 by $\sim$ twofold. Hence, $3 \mathrm{x}$-cytomix was used
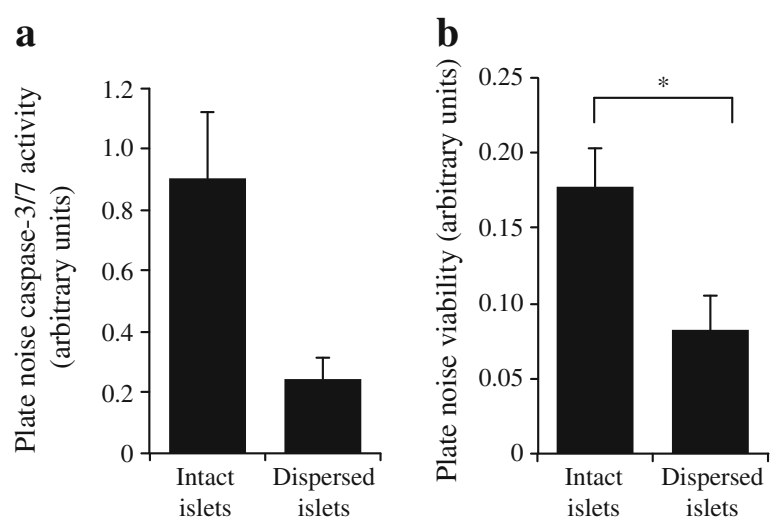

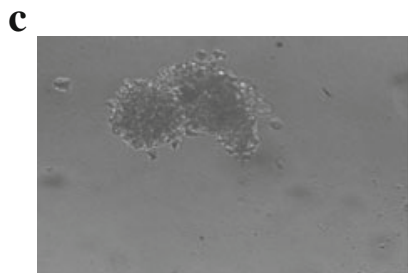

Intact islets

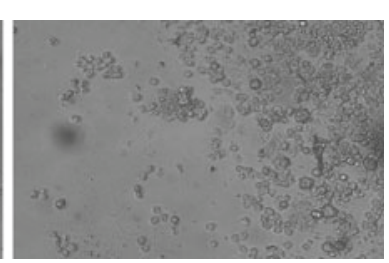

Dispersed islets

Fig. 1 Effect of dispersion of human islets on the signal-to-noise ratio of HTS. Intact or dispersed human islets were seeded in 384-well plates ( $\sim 13$ islets per well). Caspase-3/7 activity (a) or cellular reducing power, assayed by CTB fluorimetric assay (b) were measured as indicated and the plates' noise was calculated as described in the Methods. Results are means \pm SEM of two independent experiments in duplicate in (a) and four independent experiments in (b); $* p<0.05$ vs intact islets. (c) Photomicrographs of intact and dispersed human islets throughout the screens. To detect optimal conditions for knockdown efficacy, time course and dose-response experiments were performed using siRNA pools for a given control gene. Over $90 \%$ reduction in expression of the control gene, measured by qRT-PCR, was observed over the entire concentration range of siRNA used (25$100 \mathrm{nmol} / \mathrm{l})$ (ESM Fig. 1b), with gene silencing being optimal after $72 \mathrm{~h}$ (ESM Fig. 1c). To reduce off-target effects, the lowest concentration of siRNA pools that efficiently silenced their targets $(25 \mathrm{nmol} / \mathrm{l})$ was used during a $72 \mathrm{~h}$ incubation period throughout the screens.

HTS for modulators of beta cell death induced by cytokines HTS for regulators of cytokine-induced death of human beta cells were carried out using 730 human siRNA pools. These were selected based upon our previous HTS of 3,850 mouse siRNAs applied to cytokine-treated MIN6 cells [23]. siRNA libraries enriched in high-score hits in the mouse screens [23] (e.g. ubiquitin ligases, deubiquitinating enzymes) were chosen, as well as individual siRNA pools from other libraries having a $z$ score $>|1.5|$ in the mouse screen. The selected human siRNAs were screened for their effects on caspase-3/7 activity in dispersed human islets treated with cytokines (Fig. 2a). As shown in Fig. 2b, the distribution of the average $z$ scores was, in general, consistent among islets obtained from the nine different donors. Given siRNAs that either had positive $z$ scores and represent anti-apoptotic genes (blue) or those having a negative $z$ score and represent pro-apoptotic genes (red) gained similar scores (positive or negative) in most patients. Still, many $z$ score values were $<|1.0|$ (Fig. 2c), resulting in $m z$ score $=0$ (see Methods) for the majority of the siRNAs. Only a few siRNAs had $m z$ scores $>4.4$, and were considered potential (pro- or anti-apoptotic) hits (Table 1). A number of these hits, such as OTUB2, RNF40 and USP18, were not previously associated with beta cell death. Because OTUB2 had the highest $m z$ score (16.6) it was considered for further characterisation.

Effect of OTUB2 siRNAs on viability of beta cells The ability of OTUB2 to affect cellular viability was examined both in MIN6 cells and in human islets. OTUB2 siRNA decreased by $50 \%$ (Fig. 3a) and 75\% (Fig. 3b) the levels of its mRNA in MIN6 cells and human islets, respectively. This reduction resulted in $\sim 25 \%$ decrease in protein levels of OTUB2 in MIN6 cells (Fig. 3c). Silencing of OTUB2 increased by $60 \%$ (Fig. 4a) and 20\% (Fig. 4b), respectively, caspase-3/7 activity in cytokine-treated MIN6 cells and human islets when compared with cells treated with control siRNAs. Basal caspase $3 / 7$ activity also increased by $70 \%$ in human islets treated with OTUB2 siRNA (Fig. 4b) but not in MIN6 cells treated in a similar manner (Fig. 4a). FACS analysis revealed that silencing of Otub2 increased by two- 

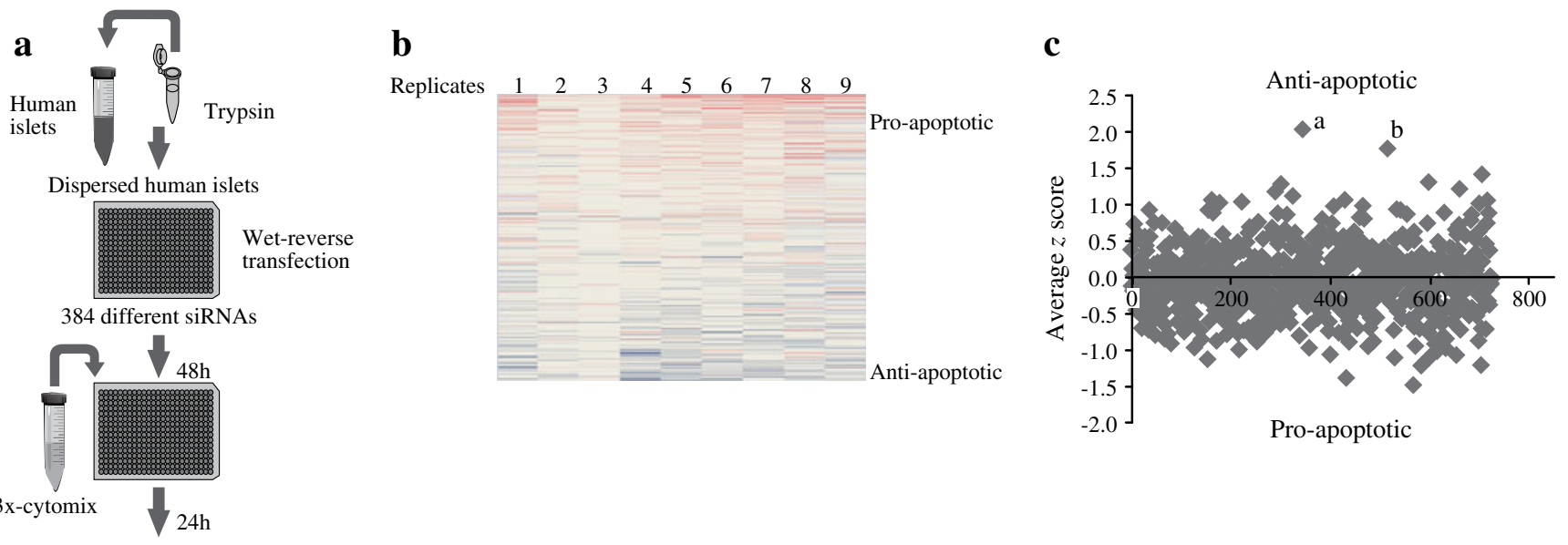

Caspase-3/7 activity detection

Fig. 2 siRNA HTS of human pancreatic islets. Cytokine-treated dispersed human islets obtained from nine donors were subjected to siRNA HTS as described in the Methods. (a) Illustration of the screen outline. (b) Distribution of average $z$ scores of the 730 siRNAs among the nine donors. Positive $z$ scores corresponding to anti-apoptotic genes are marked in blue; negative $z$ scores corresponding to pro-apoptotic genes are marked in red. (c) Plot of average $z$ scores of the 730 siRNAs that were screened. ${ }^{\mathrm{a}} O T U B 2 ;{ }^{\mathrm{b}} \mathrm{RNF} 40$
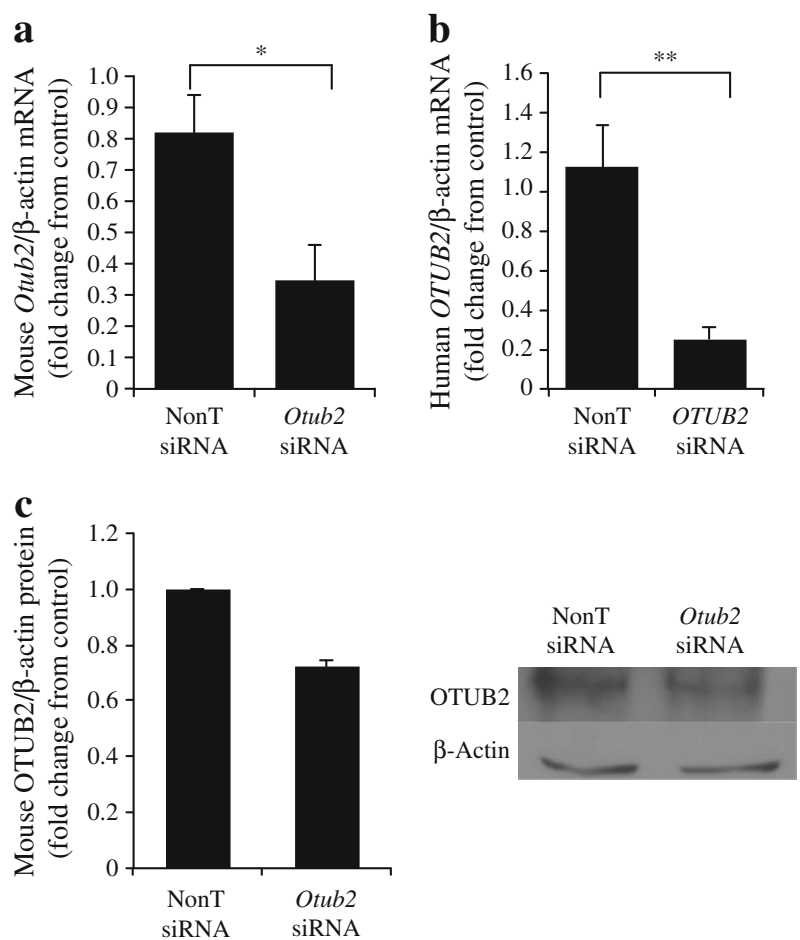

Fig. 3 Effect of OTUB2 knockdown on its mRNA and protein levels. MIN6 cells $(\mathbf{a}, \mathbf{c})$ and human islets $(\mathbf{b})$ were transfected with the indicated siRNAs for $48 \mathrm{~h}$. $(\mathbf{a}, \mathbf{b})$ Cells were harvested, total mRNA was extracted and qRT-PCR was conducted. mRNA levels were normalised to actin in the mouse samples and to GAPDH in human samples. (c) Cells were harvested, proteins were extracted and immunoprecipitated with OTUB2 antibodies. Proteins were resolved by SDS-PAGE and western blotting was conducted with the same antibodies. OTUB2 protein levels were normalised to actin levels. Data represent means \pm SEM of three independent experiments in duplicate in (a) and (b) and two independent experiments in (c); ${ }^{*} p<0.05$; ${ }^{* *} p<$ 0.01 vs NonT siRNA
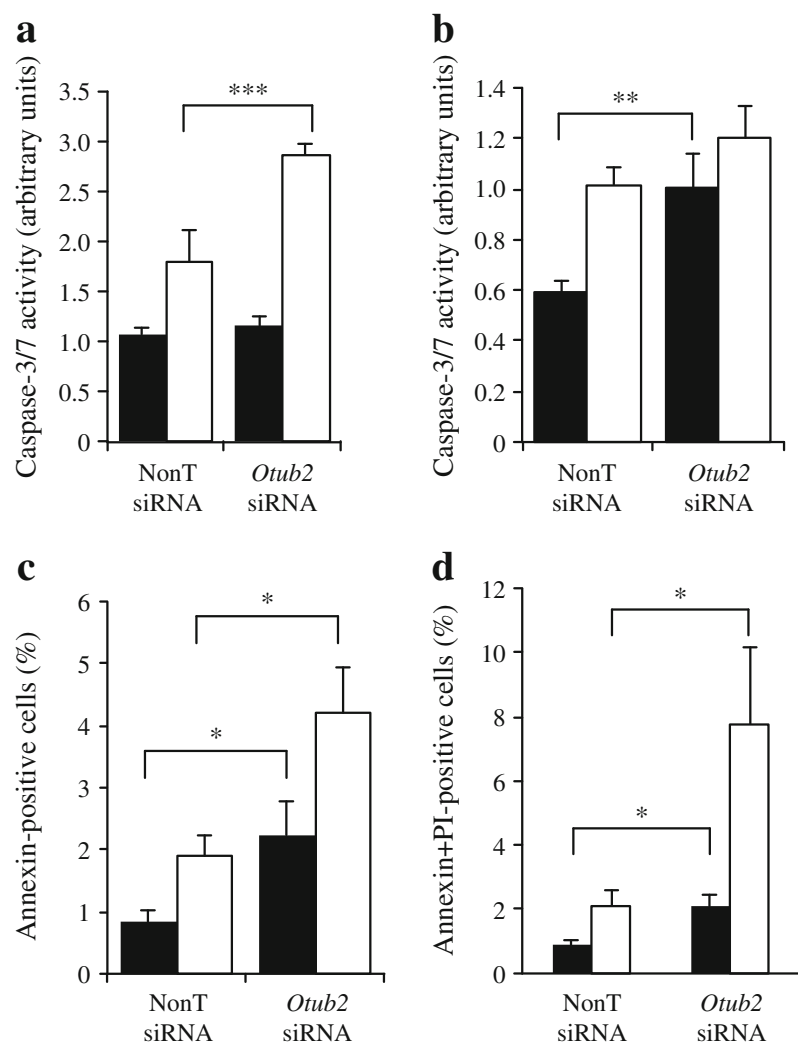

Fig. 4 Effect of OTUB2 knockdown on apoptosis and death of beta cells. MIN6 cells (a,c,d) and human islets (b) were transfected with the indicated siRNAs for $48 \mathrm{~h}$. Cells remained untreated (solid bars) or were treated with $\mathrm{x}$-cytomix for $24 \mathrm{~h}$ (open bars). Apoptosis was assayed by caspase-3/7 activity $(\mathbf{a}, \mathbf{b})$. PI and annexin-V content were determined by FACS analysis (c,d). Data represent means \pm SEM of four independent experiments in (a) and (b) and three independent experiments in five replicates in (c) and (d); ${ }^{*} p<0.05,{ }^{* *} p<0.01$ and $* * * p<0.001$ vs NonT siRNA 

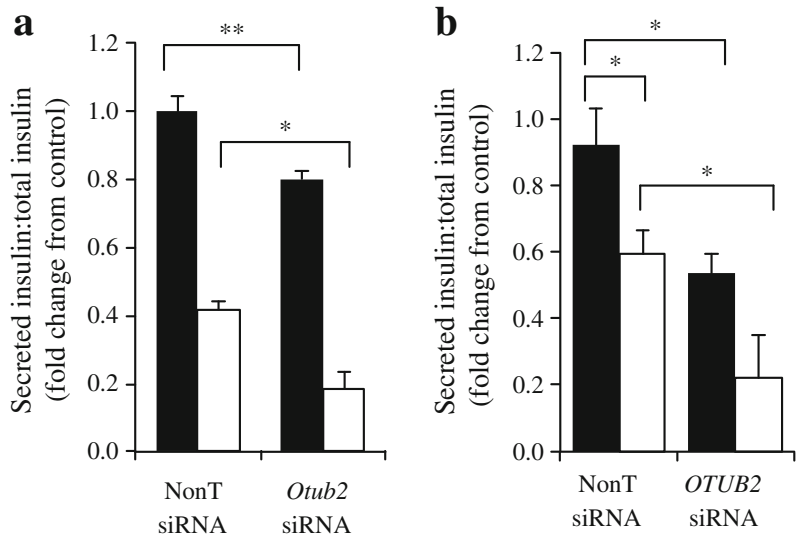

Fig. 5 Effect of OTUB2 knockdown on insulin secretion. MIN6 cells (a) and dispersed human islets (b) were transfected with the indicated siRNAs for $48 \mathrm{~h}$ and were then treated with (open bars) or without (solid bars) x-cytomix for $12 \mathrm{~h}$. GSIS was then determined as described in the Methods. Data were normalised for secretion of insulin in the absence of cytokines in cells transfected with control NonT siRNA and represent the means \pm SEM of three independent experiments; ${ }^{*} p<$ 0.05 and ${ }^{* *} p<0.01$ vs NonT siRNA

and fourfold the fraction of cytokine-induced apoptotic (Annexin-V-positive) or dead (Annexin-V+PI-positive) MIN6 cells, when compared with cells treated with control siRNAs (Fig. 4c,d). Of note, the fraction of apoptotic or dead cells under basal conditions also increased $\sim$ twofold in cells treated with Otub2 siRNA (Fig. 4c and d). These findings suggest that OTUB2 acts as a pro-survival protein in mouse and human beta cells both under basal conditions and following treatment with cytokines.

Effect of OTUB2 on insulin secretion To determine whether knockdown of OTUB2 also affects insulin secretion, glucose-stimulated insulin secretion (GSIS) was examined in MIN6 cells and intact human islets, either untreated or treated with cytokines - conditions known to inhibit GSIS [1]. As shown in Fig. 5, a $\sim 65 \%$ and $33 \%$ decrease in secreted insulin was observed when MIN6 cells or primary human islets, respectively, were treated with x-cytomix. Silencing of OTUB2 reduced GSIS by $20 \%$ and $40 \%$, respectively, already in untreated MIN6 cells or human islets, when compared with cells treated with control siRNAs. Silencing of OTUB2 further reduced GSIS by $\sim 60 \%$ when both cell types were treated with cytokines. These findings suggest that silencing of OTUB2 inhibits GSIS either in the absence or in the presence of cytokines.

Effect of OTUB2 on TRAF6 and NF- $\kappa B$ activity Cytokines are known to activate NF-KB [13], which plays a crucial role in promoting apoptosis of pancreatic beta cells [26]. Previous reports suggested that increased production of OTUB2 inhibits virus-induced activation of NF- $\mathrm{KB}$ via deubiquitination of TRAF6 and TRAF3 [22]. Indeed, the extent of ubiquitination of TRAF6, immunoprecipitated with TRAF6 antibodies, was elevated $80 \%$ in MIN6 cells treated with Otub2 siRNA, when compared with cells treated with non-targeting siRNA (Fig. 6a). As shown in Fig. 6b, the TRAF6 antibodies (but not the control IgG) selectively precipitated TRAF6 as expected. These findings support the hypothesis that de-ubiquitination of TRAF6 by OTUB2 could account for an inhibitory role played by OTUB2 on NF- $\mathrm{KB}$ signalling in beta cells.

To further address this possibility we employed a luciferase reporting system to monitor changes in NF- $\mathrm{kB}$ activity. Treatment only with cytokines increased NF-kB activity by ten-, 1.5- and eightfold, respectively, in HEK293 cells (Fig. 6c), human islets (Fig. 6d) and MIN6 cells (Fig. 6e). Knockdown of OTUB2 did not affect basal NF-kB activity in HEK293 cells (Fig. 6c) although it increased basal NF-KB activity by $\sim$ twofold in human islets (Fig. 6d) and MIN6 cells (Fig. 6e). Upon treatment with cytokines, silencing of Otub2 augmented NF- $\mathrm{KB}$ activity by twofold, $\sim 30 \%$ and $\sim 60 \%$ in HEK293, human islets and MIN6 cells, respectively. Finally, the levels of expression of NF- $\mathrm{kB}$ target genes were determined in MIN6 cells treated with Otub2 siRNAs. As shown in Table 2, the basal levels of mRNA expression of Ip-10 (also known as Cxcl10), Mcp-1 (also known as $\mathrm{Ccl} 2$ ) and $i N O S$ (also known as Nos2), three NF-kB targets [27], were increased by $\sim 4-, \sim 75$ - and $\sim 50$-fold, respectively, upon silencing of Otub2. Much higher increases were

Table 2 mRNA expression of target genes for NF- $\mathrm{KB}$

\begin{tabular}{|c|c|c|c|c|}
\hline \multirow{2}{*}{$\begin{array}{l}\mathrm{NF}-\kappa \mathrm{B} \text { target gene } \\
\text { mRNA expression/actin }\end{array}$} & \multicolumn{2}{|l|}{ Treatment } & \multicolumn{2}{|c|}{ Effect of Otub2 siRNA } \\
\hline & Control siRNA & Otub2 siRNA & Fold change & $p$ value \\
\hline Ip-10 & 1.0 & 3.9 & 3.7 & $3.6 \times 10^{-6}$ \\
\hline$M c p-1$ & 1.7 & 132 & 77 & $1.8 \times 10^{-5}$ \\
\hline$i N O S$ & 1 & 49 & 51 & 0.01 \\
\hline
\end{tabular}

Data is a representative of three independent experiments in duplicate

MIN6 cells were transfected with the indicated siRNAs for $48 \mathrm{~h}$. Cells were harvested, total mRNA was extracted and qRT-PCR was conducted. mRNA levels of the indicated genes were normalised to actin 
a

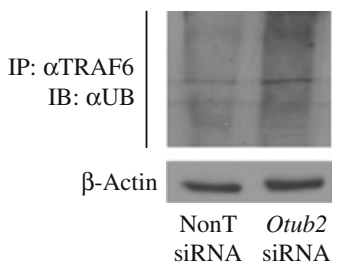

b

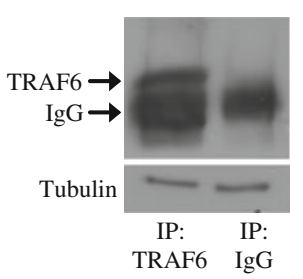

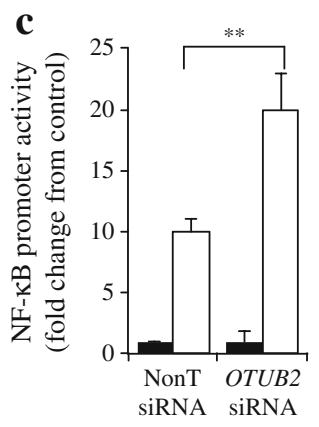

d

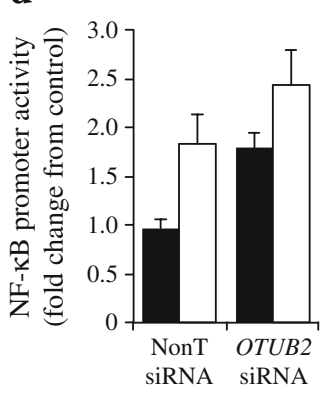

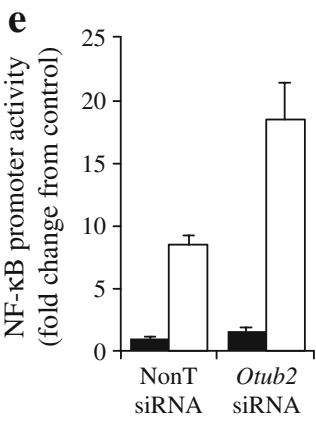

Fig. 6 Effect of OTUB2 knockdown on ubiquitination of TRAF6 and NF-KB activity. (a) MIN6 cells were transiently transfected with the indicated siRNAs. After $72 \mathrm{~h}$, cells were extracted, immunoprecipitated (IP) with anti-TRAF6 antibodies and western blotted (IB) with antiubiquitin antibodies. Blotting with actin antibodies served as control. (b), MIN6 cells were extracted, immunoprecipitated with anti-TRAF6 antibodies and western blotted with anti-TRAF6 antibodies. Blotting with tubulin antibodies served as control. (c-e) HEK293 cells (c), primary human islets (d) and MIN6 cells (e) were transiently transfected with the indicated siRNAs. At $24 \mathrm{~h}$ post siRNA transfection, cells

observed in the presence of cytokines alone, although silencing of Otub2 in the presence of cytokines only marginally increased the already highly elevated expression of these genes (data not shown). These data suggest that OTUB2 inhibits basal NF-KB activity and thus might regulate the anti-apoptotic machinery of pancreatic beta cells.

\section{Discussion}

Dispersed human pancreatic islets were used in the present study to perform functional siRNA HTS, with the aim of identifying novel genes involved in cytokine-induced beta cell death. Our findings revealed an anti-apoptotic role for OTUB2, a de-ubiquitinating enzyme that regulates the NF- $\mathrm{kB}$ signalling pathway, and demonstrated OTUB2's function in the prevention pancreatic islet death both under basal conditions and following treatment with cytokines. The use of primary human islets for functional siRNA HTS has not been previously documented and opens up a platform for new screen modalities that are of physiological significance.

A major obstacle for screening of intact primary human islets in a high-throughput manner is their large heterogeneity in size and volume, which results in a low signal-to-noise ratio and renders such a screen unfeasible. To overcome these technical limitations, dispersed human islets were used, as previously described [25]. Islet dispersion increased the yield of transfection, generated a more homogenous population and significantly increased the signal-to-noise ratio of the screen, when compared with screens we performed using intact islets. Furthermore, a means of selecting potential hits in a highly 'noisy' environment were further transfected with pNF-kB-MetLuc2 or with pMetLuc2 (control). At $24 \mathrm{~h}$ following the second transfection, cells were treated with (open bars) or without (solid bars) x-cytomix for $18 \mathrm{~h}$. NF-kB activity was monitored using the 'Ready-to-Glow' secreted luciferase reporter system (Clontech), according to the manufacturer's protocol. Data is representative of three experiments with Otub2 siRNA in (a) and of two experiments in (b). Data are means \pm SEM of three experiments in triplicates in (c), two experiments in five replicates in (d) and two experiments in three replicates in (e); ${ }^{* *} p<0.01$ vs NonT siRNA

was developed. This novel method and its analysis confers robustness combined with high physiological relevance.

Overall, 730 siRNAs were screened using dispersed islets from nine human donors. The siRNAs were selected from siRNA libraries enriched in high-score hits, revealed in our previous HTS of 3,850 siRNAs introduced into cytokine-treated MIN6 cells [23]. The implementation of such pre-selection enabled an $80 \%$ reduction in the number of siRNAs being tested in the current screen. This allowed us to repeat the screen in a larger number of donors and thus increase the validity of the results. The screen identified a number of genes (Table 1) that were not previously implicated in the regulation of beta cell death; still, all these 19 candidate genes required further validation before they could be considered as physiological regulators of beta cell survival. OTUB2 was one of the most significant potential hits revealed by the current screen. It scored highly in independent screens performed in islets derived from five (out of nine) humans, suggesting that its effects were great enough to overcome the large variance between the individual donors.

Several lines of evidence support the above conclusion. First, we showed that inhibition of OTUB2 by its siRNAs increased caspase-3/7 activity both under basal conditions and in cytokine-treated MIN6 cells and in human islets. Second, we demonstrated that siRNAs of OTUB2 impaired insulin secretion both under basal conditions and in cytokine-treated MIN6 cells or primary human islets, thus implying a beneficial role for OTUB2 in the restoration of beta cell function. Third, we showed that silencing of Otub2 evoked NF-kB promoter activity, and increased the expression of a number of NF-kB target genes including Mcp-1, Ip-10 and $i N O S$ [27].

OTUB2 belongs to the OTU family of de-ubiquitinating enzymes [22]. It contains the OTU (ovarian tumour) domain, 
Fig. 7 Mode of action of OTUB2 in beta cells. Our working hypothesis predicts that OTUB2 deubiquitinates signalling modules (e.g. TRAF3/6) along the NF-kB signalling pathway and thus prevents the apoptotic effects of this pathway in beta cells. IRAK, IL-1R-associated kinase; TRADD, TNF receptor associated death domain. The dashed lines in the scheme indicate potential effects of OTUB2 that are yet unproven

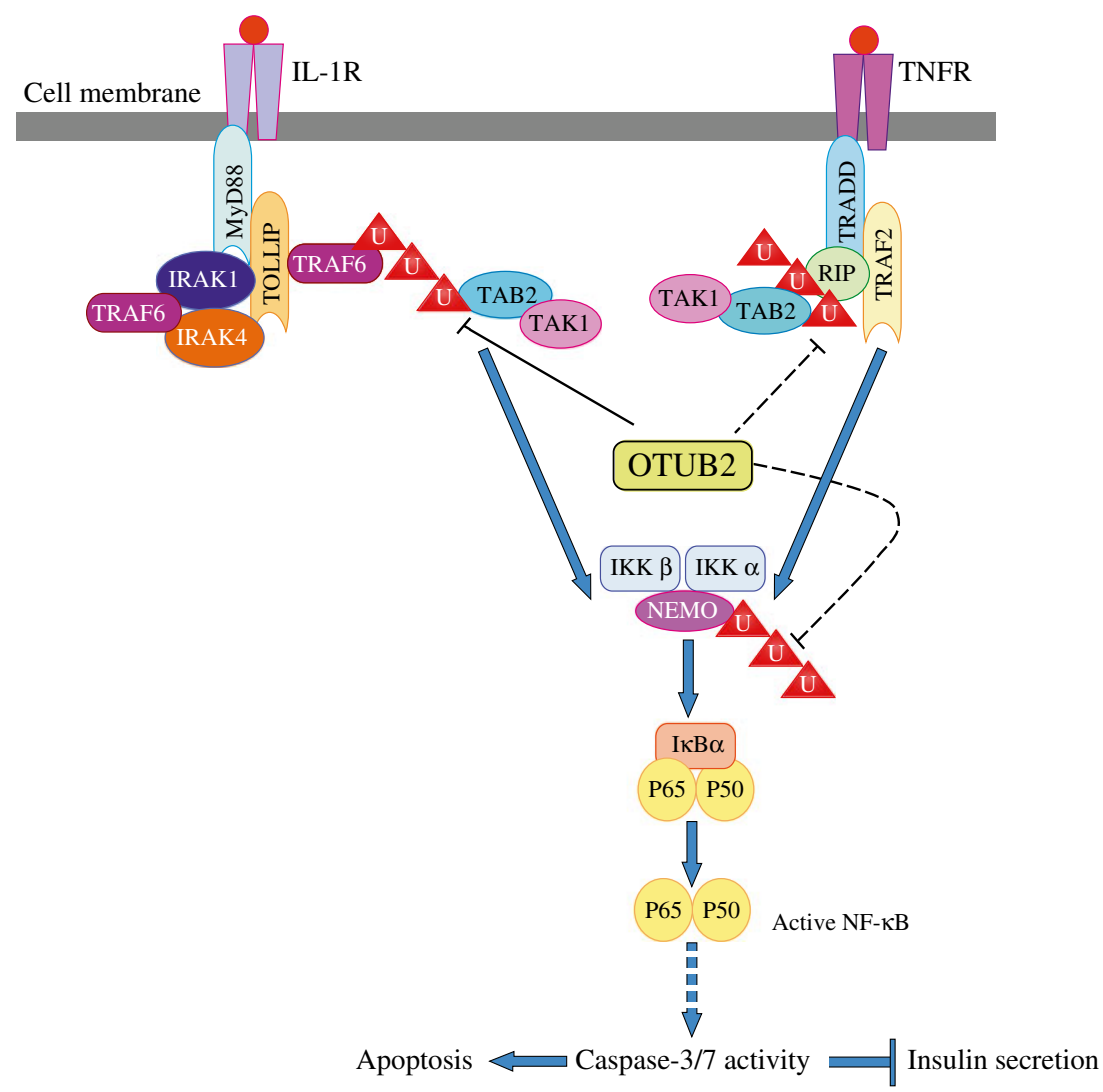

which is a conserved sequence found in viruses, bacteria, plants, yeasts and humans [28]. OTUB2 plays a role in immunity and viral infection [28] and is a negative regulator of virus-triggered type I IFN induction through deubiquitination of TRAF3 and TRAF6, leading to the inhibition of virus-induced Ifnbl gene expression and cellular antiviral responses [22]. TRAF6 is a key player in cytokine-induced NF- $\mathrm{KB}$ activation [29], therefore its deubiquitination by OTUB2 could account for the inhibitory role played by OTUB2 on NF- $\mathrm{KB}$ signalling in pancreatic beta cells. Indeed we could show that silencing of Otub2 increases the ubiquitination of TRAF6 in MIN6 cells, thus providing a potential molecular basis for the activation of NF-kB signalling in beta cells treated with Otub2 siRNAs.

OTUB2 was shown to have a clear preference for cleaving K63-linked ubiquitin [30] as opposed to its close homologue OTUB1. Polyubiquitin chains linked through K63 are assembled on cytokine receptor-interacting proteins including RIP1 and TRAF2/5 [16]. K63-polyubiquitin chains are recognised by ubiquitin-binding adaptor proteins (TGF-beta activated kinase 1 binding proteins 2/3 [TAB2/3] and NF-KB essential modulator [NEMO]) as part of the kinase complexes IKK $\alpha / \beta$ and TGF-beta activated kinase 1 (TAK1). This allows TAK1 to phosphorylate and activate IKKs. IKK $\beta$ then phosphorylates the cytoplasmic I $\kappa \mathrm{B}-\mathrm{NF}-\kappa \mathrm{B}$ complex, which triggers $\mathrm{K} 48$-linked polyubiquitination and proteasomal degradation of $\mathrm{I} \kappa \mathrm{B}$, releasing NF- $\mathrm{KB}$ to enter the nucleus and activate transcription of target genes [31]. K63 polyubiquitination also allows recruitment of the linear ubiquitination chain assembly complex [LUBAC] consisting of haem-oxidised IRP2 (iron regulatory protein 2) ubiquitin ligase-1 [HOIL-1]; HOIL-1 L interacting protein [HOIP] (also known as RNF31); and shank-associated $\mathrm{RH}$ domain interacting protein [SHARPIN], which together mediate linear ubiquitination of NEMO and RIP1 [16].

OTUB2 de-ubiquitination of K63-polyubiquitin chains could hamper NF- $\mathrm{KB}$ activation by modifying scaffold elements. We show that OTUB2 de-ubiquitinates TRAF6 and hypothesise that it may also de-ubiquitinate IKK and RIP1, critical elements along the NF- $\mathrm{KB}$ cascade, and thus inhibit propagation of NF- $\mathrm{KB}$ signalling in beta cells (see Fig. 7). Due to the pro-apoptotic role of NF- $\mathrm{KB}$ in beta cells, deubiquitination of TRAF6 and attenuation of NF- $\mathrm{KB}$ signals by OTUB2 are expected to result in beta cell survival. This hypothesis is supported by the fact that TRAF3/6 are OTUB2 targets in other cells [22], that NF- $\mathrm{BB}$ promotes beta cell mortality [32] and that OTUB2 siRNAs promote NF- $\kappa$ B activity.

In summary, our findings highlight the crucial role of OTUB2 in the inhibition of non-proteolytic ubiquitination of NF-KB signalling elements, thus attenuating the propagation of this signalling pathway and its adverse effects on human beta cells. The novel role of genes, OTUB2 included, identified by the siRNA HTS of human islets as potential 
regulators of beta cell survival, deserves further characterisation as it offers new prospects in our search for targets for therapeutic interventions in type 1 diabetes and its complications.

Acknowledgements We thank S. T. Klein (Bar Ilan University) for mathematical formulation of the data and S. Sampson (Bar Ilan University) for his critical review of the manuscript. We thank J. I. Miazaki (Osaka University, Osaka, Japan) for providing MIN6 cells. Y. Zick is the incumbent of the Marte R. Gomez Professorial Chair. Human islets were provided through the JDRF award 31-2008-413 (ECIT Islet for Basic Research program).

Funding This work was supported by grants from the JDRF International (40-2009-720) and the Israel Science Foundation (759/09).

Duality of interest The authors declare that there is no duality of interest associated with this manuscript.

Contribution statement $\mathrm{AB}, \mathrm{YV}, \mathrm{HS}-\mathrm{A}, \mathrm{RI}, \mathrm{SS}, \mathrm{GJ}$ and SB-H contributed to conception and design of the experiments, data collection, data analysis, interpretation and drafting of the article. SB-H and $\mathrm{YZ}$ contributed to conception and design of the experiments, data analysis and interpretation, drafting of the article and revising it critically for important intellectual content. All authors gave final approval of the version to be published.

\section{References}

1. Tarabra E, Pelengaris S, Khan M (2012) A simple matter of life and death - the trials of postnatal beta-cell mass regulation. Int $\mathrm{J}$ Endocrinol 1:1-2. doi:10.1155/2012/516718

2. Grieco FA, Sebastiani G, Spagnuolo I, Patti A, Dotta F (2012) Immunology in the clinic review series; focus on type 1 diabetes and viruses: how viral infections modulate beta cell function. Clin Exp Immunol 168:24-29

3. Hogan A, Pileggi A, Ricordi C (2008) Transplantation: current developments and future directions; the future of clinical islet transplantation as a cure for diabetes. Front Biosci 13:1192-1205

4. Emamaullee JA, Shapiro AM (2006) Interventional strategies to prevent beta-cell apoptosis in islet transplantation. Diabetes 55:1907-1914

5. Allagnat F, Fukaya M, Nogueira TC et al (2012) C/EBP homologous protein contributes to cytokine-induced pro-inflammatory responses and apoptosis in beta-cells. Cell Death Differ 19:1836-1846

6. Cnop M, Welsh N, Jonas JC, Jorns A, Lenzen S, Eizirik DL (2005) Mechanisms of pancreatic beta-cell death in type 1 and type 2 diabetes: many differences, few similarities. Diabetes 54(suppl 2): S97-S107

7. Araki E, Oyadomari S, Mori M (2003) Impact of endoplasmic reticulum stress pathway on pancreatic beta-cells and diabetes mellitus. Exp Biol Med (Maywood) 228:1213-1217

8. Carrington EM, McKenzie MD, Jansen E et al (2009) Islet betacells deficient in Bcl-xL develop but are abnormally sensitive to apoptotic stimuli. Diabetes 58:2316-2323

9. Bedoya FJ, Salguero-Aranda C, Cahuana GM, Tapia-Limonchi R, Soria B, Tejedo JR (2012) Regulation of pancreatic beta-cell survival by nitric oxide: clinical relevance. Islets 4:108-118

10. Kim KA, Lee MS (2009) Recent progress in research on beta-cell apoptosis by cytokines. Front Biosci 14:657-664
11. Zhao Y, Krishnamurthy B, Mollah ZU, Kay TW, Thomas HE (2011) NF-kappaB in type 1 diabetes. Inflamm Allergy Drug Targets 10:208-217

12. Sarkar SA, Kutlu B, Velmurugan K et al (2009) Cytokinemediated induction of anti-apoptotic genes that are linked to nuclear factor kappa-B (NF-kappa B) signalling in human islets and in a mouse beta cell line. Diabetologia 52:1092-1101

13. Ortis F, Miani M, Colli ML et al (2012) Differential usage of NFkappaB activating signals by IL-1 beta and TNF-alpha in pancreatic beta cells. FEBS Lett 586:984-989

14. Bravo-Egana V, Rosero S, Klein D et al (2012) Inflammationmediated regulation of microRNA expression in transplanted pancreatic islets. J Transplant 1:1-2. doi:10.1155/2012/723614

15. Chiu YH, Zhao M, Chen ZJ (2009) Ubiquitin in NF-kappaB signaling. Chem Rev 109:1549-1560

16. Verhelst K, Carpentier I, Beyaert R (2011) Regulation of TNFinduced NF-kappaB activation by different cytoplasmic ubiquitination events. Cytokine Growth Factor Rev 22:277-286

17. Chen ZJ (2005) Ubiquitin signalling in the NF-kappaB pathway. Nat Cell Biol 7:758-765

18. Ramadan N, Flockhart I, Booker M, Perrimon N, Mathey-Prevot B (2007) Design and implementation of high-throughput RNAi screens in cultured Drosophila cells. Nat Protoc 2:2245-2264

19. Whitehurst AW, Bodemann BO, Cardenas J et al (2007) Synthetic lethal screen identification of chemosensitizer loci in cancer cells. Nature 446:815-819

20. Moreau D, Kumar P, Wang SC et al (2011) Genome-wide RNAi screens identify genes required for ricin and PE intoxications. Dev Cell 21:231-244

21. Balakirev MY, Tcherniuk SO, Jaquinod M, Chroboczek J (2003) Otubains: a new family of cysteine proteases in the ubiquitin pathway. EMBO Rep 4:517-522

22. Li S, Zheng H, Mao AP et al (2010) Regulation of virus-triggered signaling by OTUB1- and OTUB2-mediated deubiquitination of TRAF3 and TRAF6. J Biol Chem 285:4291-4297

23. Beck A, Isaac R, Lavelin I et al (2011) An siRNA screen identifies transmembrane 7 superfamily member 3 (TM7SF3), a seven transmembrane orphan receptor, as an inhibitor of cytokine-induced death of pancreatic beta cells. Diabetologia 54:2845-2855

24. Boura-Halfon S, Shuster-Meiseles T, Beck A et al (2010) A novel domain mediates insulin-induced proteasomal degradation of insulin receptor substrate 1 (IRS-1). Mol Endocrinol 24:2179-2192

25. Williams SJ, Wang Q, Macgregor RR, Siahaan TJ, Stehno-Bittel L, Berkland C (2009) Adhesion of pancreatic beta cells to biopolymer films. Biopolymers 91:676-685

26. Larsen L, Storling J, Darville M et al (2005) Extracellular signalregulated kinase is essential for interleukin-1-induced and nuclear factor kappaB-mediated gene expression in insulin-producing INS1 E cells. Diabetologia 48:2582-2590

27. Baker MS, Chen X, Rotramel A, Nelson J, Kaufman DB (2003) Proinflammatory cytokines induce NF-kappaB-dependent/NO-independent chemokine gene expression in MIN6 beta cells. J Surg Res 110:295-303

28. Komander D, Barford D (2008) Structure of the A20 OTU domain and mechanistic insights into deubiquitination. Biochem J 409:77-85

29. Hostager BS (2007) Roles of TRAF6 in CD40 signaling. Immunol Res 39:105-114

30. Edelmann MJ, Iphofer A, Akutsu M et al (2009) Structural basis and specificity of human otubain 1-mediated deubiquitination. Biochem J 418:379-390

31. Adhikari A, Xu M, Chen ZJ (2007) Ubiquitin-mediated activation of TAK1 and IKK. Oncogene 26:3214-3226

32. Rink JS, Chen X, Zhang X, Kaufman DB (2012) Conditional and specific inhibition of NF-kappaB in mouse pancreatic beta cells prevents cytokine-induced deleterious effects and improves islet survival posttransplant. Surgery 151:330-339 